\title{
Thermal catalytic conversion of the used isobutyl isoprene rubber into valuable hydrocarbons
}

\author{
M. Rasul Jan · Farah Jabeen • Jasmin Shah • \\ Fazal Mabood
}

Received: 4 July 2009/Accepted: 16 October 2009/Published online: 4 November 2009

(c) The Author(s) 2009. This article is published with open access at Springerlink.com

\begin{abstract}
An environment friendly method, thermal catalytic pyrolysis of used isobutyl isoprene rubber was investigated. In this method, the used inner butyl tube rubbers were catalytically pyrolyzed into valuable hydrocarbons and carbon black. In this method, the tube rubber was pyrolyzed both thermally (with out catalyst) and catalytically in a batch reactor under atmospheric pressure. The effect of temperature, the amount of catalyst, and the reaction time on the yields of the pyrolyzed products were investigated. Char yield decreased with increase of pyrolysis temperature while total gas and liquid yields increased. The liquid fraction was obtained with boiling point up to $478 \mathrm{~K}$. At optimum conditions, the liquid product was collected and analyzed for different fuel properties. Typical analysis of the used isobutyl isoprene rubber oil for both the cases of parent and refluxed oil has been performed. Phenols and carbonyls were quantitatively determined by spectrophotometric methods using folin-denis and phenyl hydrazine reagents, respectively. The distillation data showed that $\sim 100 \%$ of oil has boiling point $<473 \mathrm{~K}$ which is the boiling point for
\end{abstract}

M. Rasul Jan

University of Malakand, Chakdara, NWFP, Pakistan

F. Jabeen $(\square)$

Department of Chemistry, Sarhad University of Science and Information Technology, Peshawar, NWFP, Pakistan

e-mail: fjabeen2009@yahoo.com

\section{J. Shah}

Institute of Chemical Sciences, University of Peshawar, Peshawar, NWFP, Pakistan

F. Mabood

Department of Chemistry, University of Malakand, Chakdara, NWFP, Pakistan
$80 \%$ of distilled product in commercial kerosene. Its specific gravity, viscosity, freezing point, Cetane number, and diesel index were also within the limits of kerosene.

Keywords Thermal and catalytic pyrolysis . Isobutyl isoprene rubber (inner butyl tube) - Liquid fuel . Zinc oxide

\section{Introduction}

Butyl rubber is a synthetic rubber with a remarkable ability to retain air. It also has resistance to deformation due to heat and weather. The combination of these factors makes it an ideal material for automobile inner tubes. The used isobutyl isoprene rubber is composed of many different components (rubbers, carbon N660, stearic acid, fillers, etc.), which are heterogeneously distributed along the tube.

The negative environmental impacts caused by disposal of waste automotive tires and the used isobutyl isoprene inner tube rubber in landfills or by incineration can be reduced by recovery of constituent chemicals and energy contents by a number of available technologies. The conventional methods for energy recovery from waste rubber were based on simple combustion in cement kilns and its use as a compound for low-value rubber goods. However, all these combined applications were not able to solve the waste rubber problems and did not result in adequate profitability.

A number of studies related to rubber pyrolysis have been reported in literature for its conversion into valuable compounds. Pyrolysis of rubber appears to be a logical choice, all the more because, apart from minor fugitive sources and equipment leaks, this process produces virtually no emissions. One of the problems with thermal 
pyrolysis is that of a wide oil product distribution with poor economical value. Therefore, catalytic pyrolysis, an alternative technique to thermal pyrolysis, is under extensive exploration. This alternative may lead to a high value hydrocarbon mixture, which could reduce the required reaction temperature, improve the yield of products, provide selectivity in the product distributions, and enhance the economic potential of the process [1-3]. William and Brindle used fixed bed [4-6] and fluidized bed [7, 8] reactors to maximize the selective determination of single ring

\section{Reaction mechanism}

Thermal pyrolysis of rubber starts with a random initiation to form polymer radicals, but the catalytic pyrolysis on solid basis is initiated by abstraction of $\mathrm{H}^{+}$from the reactants to form anionic intermediates called carboanions. The surface $\mathrm{O}^{2-}$ ions of zinc oxides abstract $\mathrm{H}^{+}$and the metal cations stabilize the carboanions. The formation of anionic species indicates the existence of an electron or electron pair donating sites on the surface.

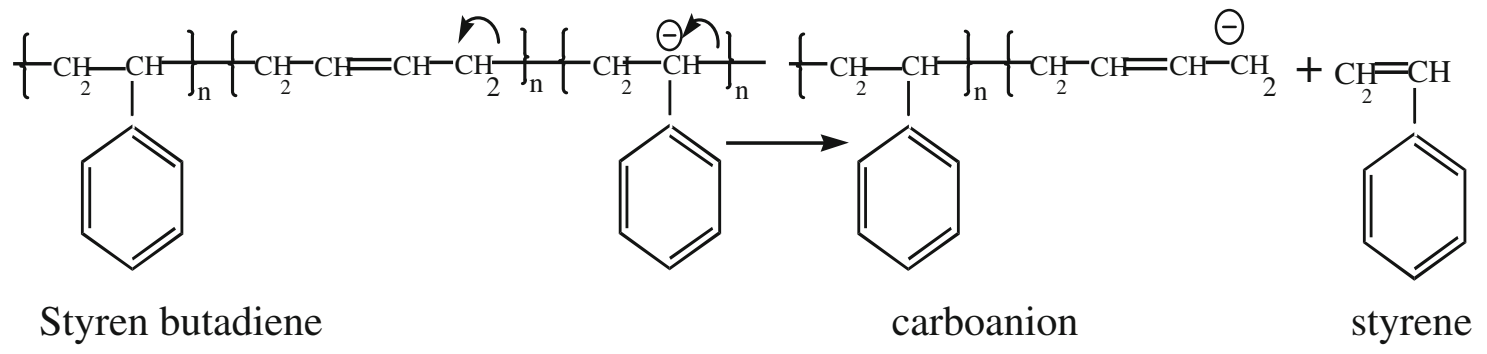

aromatic hydrocarbons. Vacuum pyrolysis experiments were carried out by Roy et al. [9], and the data obtained indicated that the light fraction of pyrolytic oil may be used as a gasoline additive, with the middle fraction as a plasticizer in rubbers and the heavy fraction for the production of good quality feedstock. Roy and Chaala [10] applied the same process using high temperature and reported high heating values for the pyrolytic gases. Similarly, Zolezzi et al. [11] used fast pyrolysis method and obtained high yield of oil with high heating values.

Fang et al. [12] studied the status of recycling of waste rubber. Various kinds of recycling approaches to waste rubber were summed up, such as reclaiming energy as fuel, reuse of the products of thermal decomposition, cleaning of leaking oil, and reuse, after simple modification, of regenerative rubber and powdered rubber.

Murata et al. [13] investigated continuous flow reactor for thermal degradation of polymers.

The objective of this study was to convert the used isobutyl isoprene rubber thermally and catalytically at relatively low temperature as well as to derive oil in the boiling range of commercial fuel oil. Therefore, for the studies reported in the literature, pyrolysis of the used isobutyl isoprene rubber has been carried out in the absence of catalyst and derived oils of high boiling point range. In this article, zinc oxide was used as a catalyst for the conversion of the used isobutyl isoprene rubber into fuel oil. Distillation data, flash point, aniline point, and other fuel properties were compared with commercial fuel oil and are in good agreement with commercial fuel oil.
In these processes, the corresponding intermediates ions are permanently regenerated, and thus they proceed by a self-propagating chain mechanism. These chains of the reaction are eventually terminated either by the recombination of free ions.

\section{Materials and methodology [14-17]}

In order to dispose of invariable and representative samples of the whole used isobutyl isoprene rubber, cross-sectional pieces of 5-10 mm width of a commercial car-used isobutyl isoprene rubbers were used for the thermal and catalytic pyrolysis experiment.

For the thermal pyrolysis, fixed amounts of used isobutyl isoprene rubber were filled into a batch reactor of glass tube. The reactor was heated from room temperature to a final temperature of $523,573,623$, and $673 \mathrm{~K}$ for $60 \mathrm{~min}$. Upon heating, however, decomposition of the used isobutyl isoprene rubber sample started. The gases released by these reactions gradually replaced the air in the reactor. The gaseous products passed through a trap, where the liquid hydrocarbon was collected. Liquid and solid (carbon black) pyrolysis yields were determined.

For the catalytic pyrolysis, fixed amounts of used isobutyl isoprene rubber pieces and catalyst (tube rubber/ $\mathrm{ZnO}$ ) were filled into a batch reactor of glass tube. The reactor was heated at 523, 573, 623, and $673 \mathrm{~K}$ for $60 \mathrm{~min}$. Liquid and solid (carbon black) pyrolysis yields were determined for each experiment by weighing the amount of 
each yield obtained and calculating the corresponding percentage. The gas yields were determined by difference.

\section{Reflux study of the parent oil derived from catalytic pyrolysis of isobutyl isoprene tube rubber using zinc oxide as catalyst}

\section{Procedure}

The parent oil product of $120 \mathrm{~mL}$, which was obtained during the catalytic pyrolysis process, and $20 \mathrm{~g}$ mass of the $\mathrm{ZnO}$ catalyst were transferred to a pyrex glass tube, and then the tube was inserted into the reactor. The temperature of the reactor was adjusted to $623 \mathrm{~K}$, and the mixture was refluxed for $2 \mathrm{~h}$. The refluxed oil was characterized using physical and chemical tests as given below.

Determination of physiochemical properties of the liquid product

The liquid column chromatography was used to separate the different groups of hydrocarbons present in the derived oil. The silica gel $60(63-200 \mu \mathrm{m}$ grain size, supplied by Merck) was packed into borosilicate glass column, and the pyrolysis oil applied to the top of the column. The column was then eluted with $n$-hexane, benzene, and methanol to produce aliphatic, aromatic, and polar fractions of the pyrolysis oil, respectively.

Fractional distillation was carried out to separate different boiling point hydrocarbon fractions from the liquid derived from catalytic pyrolysis of the used isobutyl isoprene rubber. The oil was distilled at a specific temperature until no more distilled products were collected.

The chromatographic analysis of each liquid fraction was carried out on a Shimadzu GC-14A equipped with a flame ionization detector. Separations were carried out on a fused-silica capillary column of $25 \mathrm{~m} \times 0.53 \mathrm{~mm}$ i.d with film thickness of $0.15 \mu \mathrm{m}$. The temperature was programmed from 313 to $603 \mathrm{~K}$ at a rate of $278 \mathrm{~K} / \mathrm{min}$. The detector and injector temperatures were 623 and $573 \mathrm{~K}$, respectively.

Infrared spectroscopy was used for identification of liquid product measuring absorption from 400 to $4400 \mathrm{~cm}^{-1}$.

Phenols and carbonyls were quantitatively determined by spectrophotometric methods using Folin-Denis and phenylhydrazine reagents, respectively.

Flash points was determined by Cleveland open cup method IP-36/84 and ASTM-D92-78, Density IP-59/82, API gravity IP-160/87 and ASTM-D1298-85, Kinematic viscosity IP-711/87 and ASTM-D445-87, Distillation IP-191/83 and ASTM-D216-77, and cetane index of fuel
IP-364/84 and ASTM-D976-80 were determined according to IP and ASTM standard methods for fuel.

\section{Results and discussion}

Products' yields

The solid, liquid, and gas yields (mass $\%$ ) obtained in the used isobutyl isoprene rubber thermal pyrolysis (without catalyst) experiments carried out from 523 to $673 \mathrm{~K}$ are presented in Fig. 1. It can be seen that at 523 and $573 \mathrm{~K}$ thermal pyrolysis was incomplete since only gas yields were produced in greater amount than liquid and solid. With increase of temperature from 623 to $673 \mathrm{~K}$, the $\%$ mass of gas increased from 6.8 to $30.0 \%$. This increase is due to stronger thermal pyrolysis at higher temperature.

Concerning liquid yield, there was no production of liquid oil up to $573 \mathrm{~K}$, then, it increased with temperature from 623 to $673 \mathrm{~K}$. The $\%$ mass of liquid increased from 6.07 to $22.23 \%$.

Concerning solid yield, up to $623 \mathrm{~K}$, there was no response of formation of carbon black, as the residue was in semi-solid state. The solid yield produced at $673 \mathrm{~K}$, at which temperature the \% mass of solid was $45.87 \%$.

The solid, liquid, and gas yields (mass\%) obtained in the used isobutyl isoprene rubber catalytic pyrolysis $(\mathrm{ZnO}$ catalyst) experiments carried out from 523 to $673 \mathrm{~K}$ are presented in Fig. 2. It can be seen that at 526, 573, and $623 \mathrm{~K}$, catalytic pyrolysis was incomplete since solid yields were much higher than the theoretical value of carbon present. With increase of temperature from 623 to $673 \mathrm{~K}$, the $\%$ mass of char decreased to $39.66 \%$, almost equivalent at $623 \mathrm{~K}$ to $673 \mathrm{~K}$ but somewhat higher than the theoretical yield $31 \%$. Therefore, one might think that not all the volatile matter of the used isobutyl isoprene rubber has been decomposed to liquid and gases. However, it has been reported in the literature $[18,19]$ that a certain amount

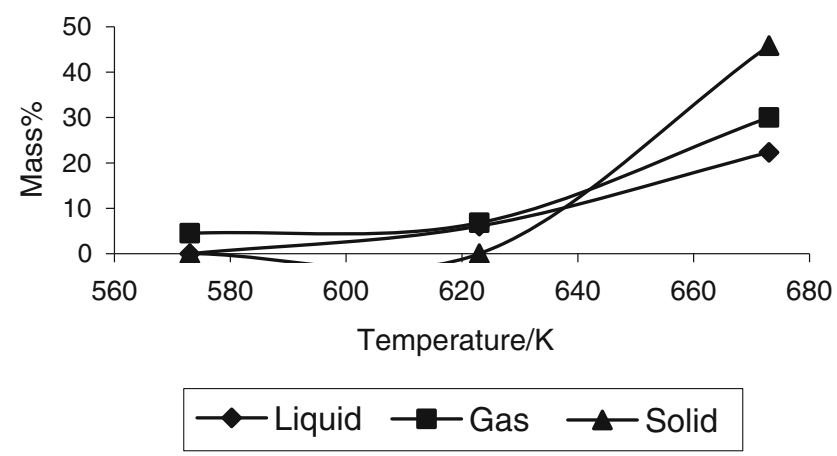

Fig. 1 Temperature optimization for thermal pyrolysis of the used butyl rubber 


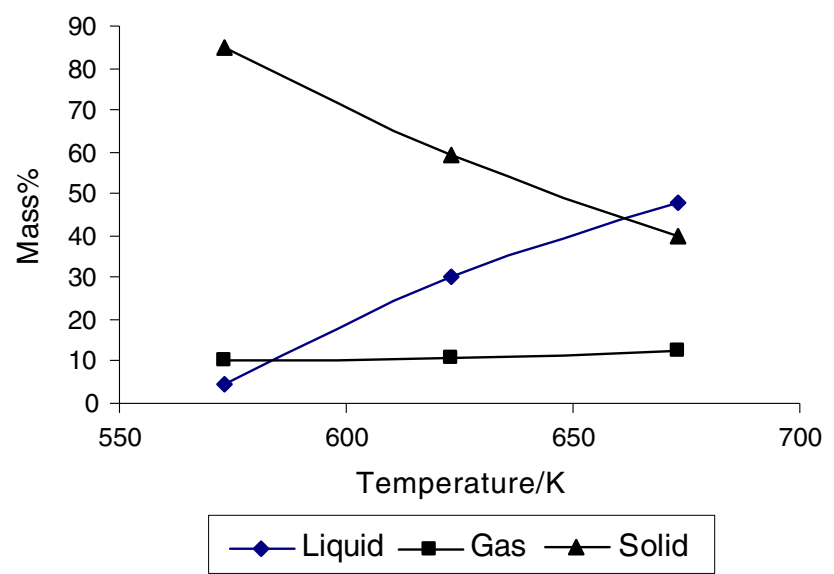

Fig. 2 Temperature optimization of butyl tube rubber catalytic pyrolysis

of char or coke-like carboneous material is formed in the pyrolysis of many polymeric materials.

Concerning liquid yield, it increased with temperature from 523 to $623 \mathrm{~K}$ and at $673 \mathrm{~K}$, there was increase in liquid due to stronger thermal pyrolysis at higher temperature; the \% mass of liquid increased from 4.67 to $47.61 \%$. The gas yield increased with increase in temperature from 523 to $673 \mathrm{~K}$; \% mass of gas increased from 10.46 to $12.74 \%$. Therefore, concerning catalytic pyrolysis yields, it can be concluded that $673 \mathrm{~K}$ is the optimum temperature for recycling the used isobutyl isoprene rubber under catalytic pyrolysis. Below this temperature $(673 \mathrm{~K})$, the conversion into liquid oil was minimum, while above $673 \mathrm{~K}$ the conversion into gaseous product was high because at high temperature, the cracking process speeds up, and results in the formation of gaseous product.

The effect of catalyst mass on products yields was also studied in the range of 0 to $2 \mathrm{~g}$ (Fig. 3). In the absence of

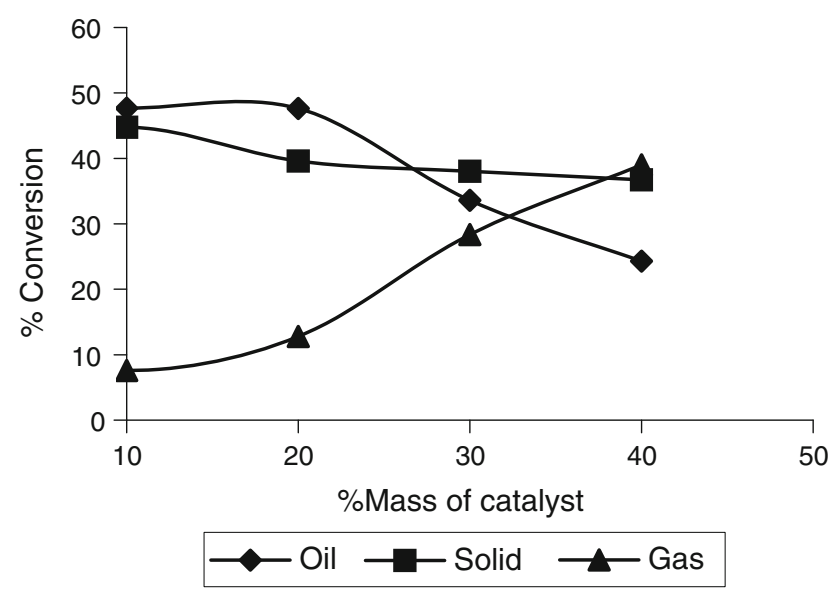

Fig. 3 Catalyst mass optimization for catalytic pyrolysis of the used butyl rubber catalyst, the conversion into liquid and gas was only $26.61 \%$ and $27.74 \%$, respectively. While in the presence of catalyst, the yield of liquid increased up to $47.64 \%$ with $0.5 \mathrm{~g}$ mass of catalyst, that of char and gas decreased to 44.76 and $7.6 \%$, respectively. As the mass of catalyst increased, the conversion into liquid product decreases, because at high catalyst mass, the cracking process speeds up. It results in the formation of gaseous product. This is because the rate of reaction increases the cracking process, which result in maximum gaseous product.

Figure 4 shows the results obtained with inner butyl rubber catalytic pyrolysis as a function of time, operating at $673 \mathrm{~K}$. The inner butyl rubber is completely converted into THF soluble products in a reaction time of $60 \mathrm{~min}$ because the same values for total conversion are found at longer reaction times, and in addition, these values are the maximum. The conversion into liquid and gas was 47.60 and $7.75 \%$, respectively. Below this reaction time, the conversion into liquid oil was minimum, and the residue was high, while above this condition, there is no increase in the yield of liquid oil. This is because the whole product was converted into oil and gas product, and the remaining residue was carbon black, which could not be further converted.

At optimum conditions, the liquid product was collected and analyzed for different fuel properties.

\section{Used isobutyl isoprene rubber liquid}

The liquid obtained from thermal catalytic liquefaction of isobutyl isoprene rubber is a brown color product, usually termed oils. In order to better analyze the potential uses of used isobutyl isoprene rubber oils, distillation test at atmospheric pressure was carried out, at optimum conditions. The results are presented in Fig. 5. It was observed that $60 \%$ volume of such oils was easily distillable fraction with

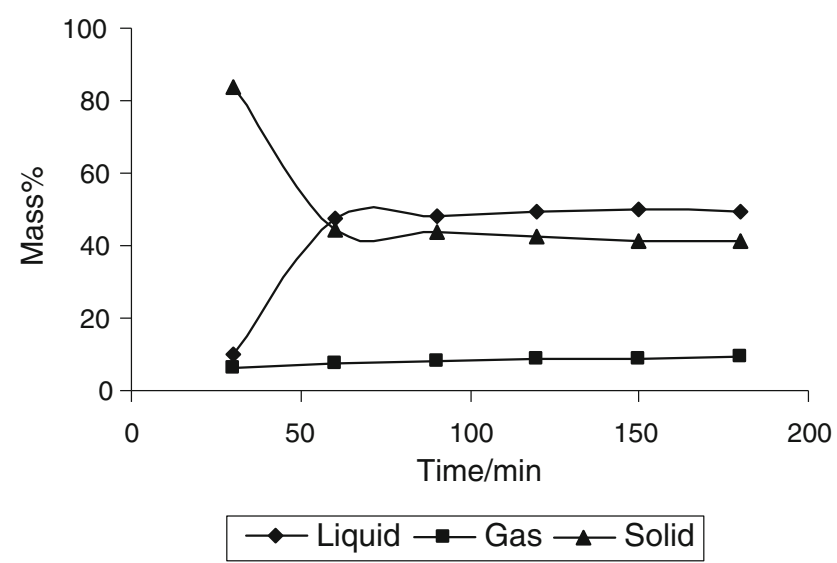

Fig. 4 Time optimization for catalytic pyrolysis of the used isobutyl rubber 


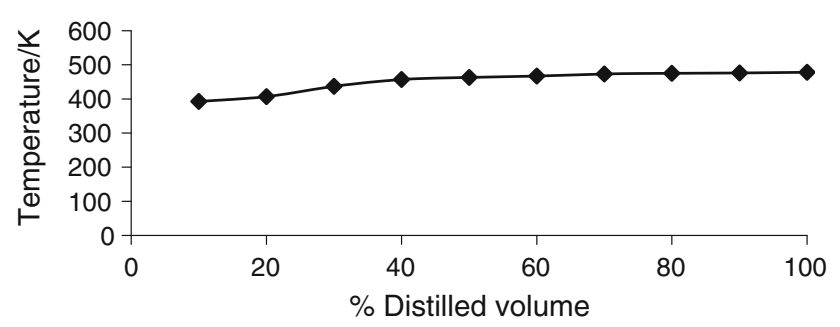

Fig. 5 Distillation of catalytic pyrolysis of the used isobutyl tube rubber

boiling temperature ranging between $363 \mathrm{~K}$ and $497 \mathrm{~K}$, which is the boiling point range specified for kerosene.

On the contrary, Fig. 5 shows that dew point of the used isobutyl isoprene rubber oil has a boiling point $<363 \mathrm{~K}$, which is the boiling temperature for the $50 \%$ of distilled product in commercial gasoline.

This shows that the used isobutyl isoprene rubber oil fraction has more light products than the commercial gasoline oil and kerosene in the temperature range from 363 to $493 \mathrm{~K}$. The advantage is that the used isobutyl isoprene rubber oil can be atomized well, and it is possible to initiate combustion at a lower temperature. In order to establish the real potential use of such used isobutyl isoprene rubber oil fraction as gasoline and kerosene oil, a more thorough characterization was performed.

Separation of oil on silica gel column and analysis on GC were carried out with the aim of getting an idea of the nature and type of compounds present in such oil. The sample was loaded onto the column, and elution was done using different solvents like $n$-hexane, benzene, and methanol. It was expected that aliphatic fraction will be eluted with $n$-hexane, aromatic with benzene, and polar hydrocarbons with methanol. The results are given in Table 1 . It can be seen from these results that the derived oil has maximum fraction of non-polar (40\%) followed by relatively non-polar (30\%), and minimum polar fraction $(25 \%)$.

The oxygenated compounds such as phenols and carbonyls were determined quantitatively in the polar fraction of the oil sample by spectrophotometric method, the results of which are given in Table 2. The presence of these compounds may be explained by the pyrolysis of oxygenated compounds of the butyl tube rubber, such as stearic acid, etc. [20, 21].

Table 1 Percentage mass of hydrocarbon group types in the oil derived from the catalytic pyrolysis of the used butyl tube

\begin{tabular}{ll}
\hline $\begin{array}{l}\text { Hydrocarbon } \\
\text { Group }\end{array}$ & $\begin{array}{l}\text { Oil derived } \\
\text { with } \mathrm{ZnO}(\%)\end{array}$ \\
\hline Aliphatic & 40 \\
Aromatic & 25 \\
Polar & 30 \\
\hline
\end{tabular}

Table 2 Percentage mass of phenols and carbonyls in the oil derived from the catalytic pyrolysis of the used butyl tube rubber

\begin{tabular}{lll}
\hline & $\begin{array}{l}\text { Oil derived } \\
\text { with ZnO }\end{array}$ & $\begin{array}{l}\text { Refluxed } \\
\text { oil }\end{array}$ \\
\hline Phenols (ppm) & 760 & 780 \\
Carbonyls (\%) & 0.0178 & 0.0188 \\
\hline
\end{tabular}

Table 3 Typical properties of oil derived from catalytic pyrolysis of butyl tube rubber parent oil and refluxed oil

\begin{tabular}{llll}
\hline S. No. & Parameters & $\begin{array}{l}\text { Parent oil } \\
\text { of tube rubber }\end{array}$ & $\begin{array}{l}\text { Refluxed } \\
\text { oil }\end{array}$ \\
\hline 1 & Density (g/mL) & 0.7854 & 0.7985 \\
2 & Specific gravity & 0.79068 & 0.80386 \\
3 & API gravity & 47.39 & 45.71 \\
4 & Viscosity (Centipois) & 1.2999 & 1.4397 \\
6 & Kinematic viscosity $\left(\mathrm{mm}^{2} / \mathrm{s}\right)$ & 1.551 & 1.803 \\
7 & Aniline point/K & 344 & 335 \\
8 & Flash point/K & 317 & 313 \\
9 & Watson characterization constant & 11.82 & 11.721 \\
10 & Freezing point/K & 259 & 260 \\
11 & Cetane number & 51 & 51 \\
12 & Sulphur (\%) & 0.55 & 0.56 \\
13 & Refractive index & 1.4665 & 1.4555 \\
\hline
\end{tabular}

Typical analysis of the used isobutyl isoprene rubber oil for both the parent and refluxed oil in each case has been measured and are presented in Table 3. The fuel properties such as density, specific gravity, API gravity, viscosity, Kinematic viscosity, aniline point, flash point, Watson characterization constant, freezing point, diesel index, sulfur, and ASTM distillation were compared with standard values for gasoline, kerosene, and diesel oil [22]. It can be seen that the densities, API gravities, viscosities, Kinematic viscosities of both parent and refluxed oil lies in the range of kerosene.

The specific gravity of parent oil lies in the range of kerosene while that of refluxed oil lies in the range of diesel oil. Aniline point of parent oil lies in the range of diesel while that of refluxed oil lies in the range of kerosene oil. Diesel index of parent oil lies in the range of gasoline while that of refluxed oil lies in the range of kerosene oil. Flash point of used isobutyl isoprene rubber oil is within the range of flash points specified for commercial gasoline and kerosene. Other parameters such as freezing point, cetane number, and Watson characterization constant were also within the limits of diesel fuel. Therefore, the used isobutyl isoprene rubber oils, as a whole, can be considered rather good quality liquid fuels with a potential use similar to kerosene, gasoline, and commercial diesel oil. 


\section{Conclusions}

Catalytic pyrolysis of the used isobutyl isoprene rubber is the best method for maximum conversion into useful product, and this method has several advantages over other alternative recycling methods used for the used isobutyl isoprene rubber. This is because in the case of thermal analysis, the \% conversion into useful product is less and the temperature needed is high. As far as the catalyst is concerned, $\mathrm{ZnO}$ was found to be the most effective catalyst on the basis of good \% yield into liquid oil at low temperature. It has been shown that the conversion at lower temperature in the presence of zinc oxide as catalyst for the used isobutyl isoprene rubber into liquid is a feasible process. An important difference is that the oil obtained has relatively low boiling range with pyrolysis in the presence of zinc oxide catalyst as compared to that in the absence of catalyst. From spectrometric study, it was found that thermal catalytic pyrolysis of tube rubber leads to the formation of complex mixture of alkanes, alkenes, carbonyl group-containing compounds such as aldehydes, ketones, ethers, aromatic compounds such as benzene, toluene, xylene, cresol etc. The total pyrolytic oil may be directly used as fuel oil.

Open Access This article is distributed under the terms of the Creative Commons Attribution Noncommercial License which permits any noncommercial use, distribution, and reproduction in any medium, provided the original author(s) and source are credited.

\section{References}

1. Rodriguez IM, Laresgoiti MF, Cabrero MA, Torres A, Chomon MJ, Caballero B. Pyrolysis of scrap tyres. Fuel Process Technol. 2001;72:9-22.

2. Chen F, Qian J. Studies of the thermal degradation of waste rubber. J Waste Manag. 2003;23:463-7.

3. Lagrega MD, Buckingum PL, Evans JC. Hazardous waste management. New York: McGraw-Hill, Inc.; 1994.

4. William PT, Brindle AJ. Aromatic chemicals from the catalytic pyrolysis of scrap tyres. J Anal Appl Pyrolysis. 2003;67:143-64.
5. William PT, Brindle AJ. Temperature selective condensation of tyre pyrolysis oils to maximise the recovery of single ring aromatic compounds. Fuel. 2003;82:1023-31.

6. William PT, Brindle AJ. Catalytic pyrolysis of tyres: influence of catalyst temperature. Fuel. 2002;81:2425-34.

7. William PT, Brindle AJ. Fluidised bed catalytic pyrolysis of scrap tyres: influence of catalyst: tyre ratio and catalyst temperature. Waste Manag Res. 2002;20:546-55.

8. William PT, Brindle AJ. Fluidised bed pyrolysis and catalytic pyrolysis of scrap tyres. Environ Technol. 2003;24:921-9.

9. Roy C, Chaala A, Darmstadt H. The vacuum pyrolysis of used tires: end-uses for oil and carbon black products. J Anal Appl Pyrolysis. 1999;51:201-21.

10. Roy C, Chaala A. Vacuum pyrolysis of automobile shredder residues. Resour Conserv Recycl. 2001;32:1-27.

11. Zolezzi M, Nicolella C, Ferrara S, Iacobucci C, Rovatti M. Conventional and fast pyrolysis of automobile shredder residues (ASR). Waste Manag. 2004;24:691-9.

12. Fang Y, Zhan M, Wang Y. The status of recycling of waste rubber. Mater Design. 2001;22:123-8.

13. Murata K, Hirano Y, Sakata Y, Uddin MA. Basic study on a continuous flow reactor for thermal degradation of polymers. J Anal Appl Pyrolysis. 2002;65:71-90.

14. Shah J, Jan MR, Mabood F, Shahid M. Preparation and characterization of carbon black from waste tyres and their utilizations as adsorbent. J Chin Chem Soc. 2006;53:1085-9.

15. Shah J, Jan MR, Mabood F. Catalytic conversion of waste tyres into valuable liquid fuels. J Polym Environ. 2007;15:207-11.

16. Shah J, Jan MR, Mabood F. Conversion of waste tires into liquid hydrocarbons via base catalysis. Iran J Chem Chem Eng. 2008;27:103-9.

17. Shah J, Jan MR, Mabood F. Recovery of value-added product from the catalytic pyrolysis of waste tyre. Energy Convers Manag. 2009;50:991-4.

18. Grittner N, Kaminsky W, Obst G. Fluid bed pyrolysis of anhydride-hardened epoxy resins and polyether-polyurethane by the Hamburg process. J Anal Appl Pyrolysis. 1993;25:293-9.

19. Kaminsky W. Chemical recycling of mixed plastics of pyrolysis. Adv Polym Technol. 1995;14:337-44.

20. Laresgoiti MF, Caballero BM, De Macro I, Torres A, Cabrero MA, Chomon MJ. Characterization of the liquid products obtained in tyre pyrolysis. J Anal Appl Pyrolysis. 2004;71:91734.

21. Pakdel H, Roy C, Chabot S, Poirier MA. Characterization of pyrolytic light naphtha from vacuum pyrolysis of used tyres comparison with petroleum naphtha. Fuel. 1995;74:1589-94.

22. Hobson GD. Modern petroleum technology. 4th ed. England: Applied Science Publishers Ltd; 1973. 